\title{
Refined system parameters for the pre-cataclysmic binary NN Serpentis ${ }^{\star}$
}

\begin{abstract}
R. Haefner, A. Fiedler, K. Butler, and H. Barwig
Universitäts-Sternwarte München, Scheinerstr. 1, 81679 München, Germany

e-mail: haefner@usm.uni-muenchen.de

Received 3 June 2004 / Accepted 6 August 2004

Abstract. NN Ser is known to be a 17 mag pre-cataclysmic binary consisting of a hot white dwarf and a cool late type star orbiting each other with a period of $3^{\mathrm{h}} 7^{\mathrm{m}}$. The system shows very deep eclipses and a pronounced reflection effect. Using the FORS instruments at the VLT the late type star could now be detected photometrically at 22.8 mag during eclipse and monitored spectroscopically. These data combined with earlier high speed photometric and phase-resolved spectroscopic observations form the basis for a determination of refined system parameters for NN Ser. The spectral type of the late type star is found to be M4.75. A model atmosphere analysis of the white dwarf yields a temperature of $57000 \pm 3000 \mathrm{~K}$ and $\log g=7.6 \pm 0.1$. The presence of $\mathrm{He}$ in the atmosphere $\left(N_{\mathrm{He}}=2 \pm 0.5 \times 10^{-4}\right.$ by number $)$ indicates that the white dwarf is a hydrogen-helium hybrid star of type DAO1. Since the derived radial velocity curves prevent an unambiguous determination of the mass ratio the white dwarf's mass of $0.54 \pm 0.05 M_{\odot}$ is inferred using the results of the model atmosphere analysis and recent evolutionary models. The mass of the $\mathrm{M}$ star is determined via a well calibrated M-R relation to be $0.150 \pm 0.008 M_{\odot}$. The photometric measurements are analysed using a sophisticated light curve synthesis program and yield the following results: $i=84.6^{\circ} \pm 1.1^{\circ}$, $R_{\mathrm{wd}}=0.0189 \pm 0.0010 R_{\odot}$, and $R_{\mathrm{Mstar}}$ (polar) $=0.174 \pm 0.009 R_{\odot}$. The shape of the cool star turns out to be slightly ellipsoidal. Its temperature at the un-heated hemisphere (backside) is $2920 \pm 70 \mathrm{~K}$ while the heated hemisphere (sub-stellar point) has a temperature of $7125 \pm 200 \mathrm{~K}$.
\end{abstract}

Key words. stars: binaries: eclipsing - stars: binaries: spectroscopic - stars: late-type - stars: white dwarfs stars: fundamental parameters - stars: individual: NN Ser

\section{Introduction}

The binary nature of NN Ser (PG 1550+131) was discovered by Haefner (1989). He presented a light curve (integral light) of this 17 mag object showing sine-shaped variations $(\sim 0.6$ mag) and very deep eclipses ( $>4.8 \mathrm{mag}$ ) occurring half a period after maximum light and repeating with a period of $3^{\mathrm{h}} 7^{\mathrm{m}}$. Due to the faintness of the system during eclipse the true depth could not be measured. No secondary eclipse was detectable. Two spectra were also obtained, one near maximum and the other near minimum light, showing Balmer and HeI emissions disappearing near primary eclipse. A preliminary analysis revealed that NN Ser is a detached precursor of a cataclysmic binary. Its hot white dwarf heats the facing side of the companion, a cool $\mathrm{M}$ star, thus giving rise to a strong heating effect and the formation of emission lines seen best at maximum light and disappearing near primary eclipse due to the changing aspect. Limits for the system parameters were also given. IUE spectra were used by Wood \& Marsh (1991) mainly to model the reflection effect and to determine the white dwarf temperature. Catalán et al. (1994) used phase-resolved blue and far red spectra to derive radial velocities and to provide an improved

* Based on observations collected at the European Southern Observatory (La Silla and Paranal)/Chile and at the German-Spanish Astronomical Center Calar Alto/Spain. complete set of system parameters. Finally, Pigulski \& Michalska (2002) presented new photometric data and gave an improved ephemeris.

All studies so far performed have been hampered by the fact that the true depth of the eclipse and the duration of the totality (if any), i.e. the inner contact phases of the white dwarf, were unknown. This prevented the exact determination of the radii of the components. In the past, several attempts by the authors failed to obtain this crucial information with telescopes of the $4 \mathrm{~m}$-class using sophisticated observing methods and/or a special photometer. Now, the powerful combination of the VLT and the multi-mode FORS instruments allowed a successful recording of the complete eclipse and, moreover, spectroscopy of the M star during eclipse. Details of these observations are given by Haefner (2000). The VLT data together with earlier spectroscopy and photometry form the basis for the analysis performed in this paper aimed at a redetermination of all system parameters of NN Ser.

\section{Observations and reduction}

\subsection{Photometry}

High time resolution photometric measurements were performed in June 1989 at the ESO $3.6 \mathrm{~m}$ telescope equipped with 

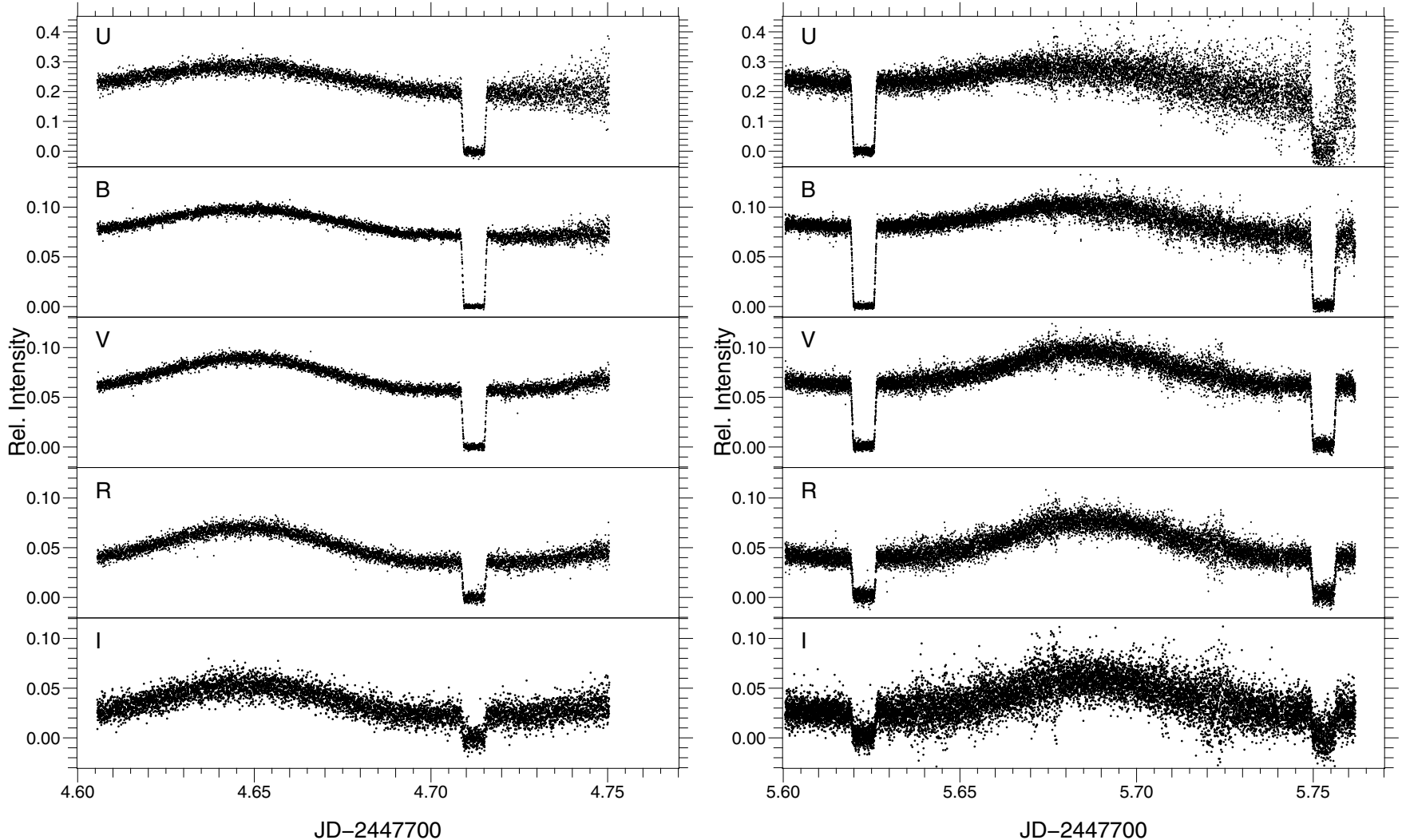

Fig. 1. Light curves of NN Ser obtained with the MCCP photometer on 1989 June 27 (left) and 28 (right). Given are intensities relative to a nearby anonymous comparison star. The larger scatter in $U$ and $I$ is due to the lower quantum efficiency of the detectors in these colour bands. The overall enhanced scatter in the light curves of 1989 June 28 is caused by unfavourable meteorological conditions during that night and the higher time resolution applied. The measurements at the end of both runs were obtained through high air mass (up to 3.5 (left) and 3.7 (right)) thus producing the increased scatter especially in the $U$ band. N.B.: The late type star is not detected during the eclipses, the recorded intensities are actually zero. Further, no secondary eclipse is noticeable.

the MCCP photometer. The MCCP (Barwig et al. 1987) allows simultaneous high-speed photometry of a target star, a comparison star and the sky in the spectral bands $U B V R I$. Details of the observations are given in Table 1. Unfortunately, the measurements of June 26 and 28 were influenced by strong cirrus which increased the scatter. Nevertheless, the concept of the MCCP yields reliable results even under such conditions. The secondary star was not detected during the five eclipses recorded. Figure 1 presents the light curves obtained on June 27 and 28. The amplitudes of the brightness variations are $0.376 \pm$ $0.005 \mathrm{mag}$ in $U, 0.332 \pm 0.002 \mathrm{mag}$ in $B, 0.487 \pm 0.004 \mathrm{mag}$ in $V, 0.722 \pm 0.008 \mathrm{mag}$ in $R$, and $0.898 \pm 0.020 \mathrm{mag}$ in $I$. Since small night to night variations of the system brightness seemed to be present an attempt was made to quantify this behaviour by normalizing all light curves to the brightness of the descending branch of the June 27 light curve. The variations turned out to be of the order of $\leq 0.1 \mathrm{mag}$ in all colours. We attribute this behaviour to the anonymous comparison star which obviously exhibits slight variability with a period of some days. However, its influence on an individual light curve is estimated to be less than $0.01 \mathrm{mag}$. An additional short observing run of $1 \mathrm{~h}$ duration was obtained in July 1998 using the MCCP at the $2.2 \mathrm{~m}$ telescope of the Calar Alto Observatory to check the accuracy of the ephemeris for the envisaged eclipse observations at the VLT.
Table 1. Journal of photometric observations.

\begin{tabular}{cccccc}
\hline \hline Date & $\begin{array}{c}\text { Start } \\
(\mathrm{UT})\end{array}$ & $\begin{array}{c}\text { Dur. } \\
(\mathrm{h})\end{array}$ & $\begin{array}{c}\text { Int. time } \\
(\mathrm{s})\end{array}$ & Filter & $\begin{array}{c}\text { No. of } \\
\text { eclipses }\end{array}$ \\
\hline 1989 June 26 & $0: 00: 00$ & $5: 10$ & 2 & $U B V R I$ & 2 \\
1989 June 27 & $2: 40: 00$ & $3: 28$ & 2 & $U B V R I$ & 1 \\
1989 June 28 & $2: 25: 00$ & $3: 55$ & 1 & $U B V R I$ & 2 \\
1998 July 12 & $0: 22: 10$ & $1: 00$ & 2 & $U B V R I$ & 1 \\
1999 June 11 & $4: 53: 05$ & $0: 19$ & trail & $V$ & 1 \\
\hline
\end{tabular}

These VLT measurements took place in June 1999 using FORS1 (see e.g. Appenzeller et al. 1998) on the first VLT $8.2 \mathrm{~m}$ Unit Telescope (ANTU). The method of observation employed was the trailing method, where the telescope performs a controlled continuous motion thus registering the light of all targets in the field of view along lines on the same frame. The drift rate was fixed at 1 pix $(24 \mu \mathrm{m}, 0.20 \operatorname{arcsec})$ per $3 \mathrm{~s}$, a compromise between the presumed integration time and the desired time resolution. Figure 2 shows the eclipse light curve as extracted from the resulting trail of NN Ser. The recorded signal during eclipse is measurable and amounts to $87 \pm 17$ counts/pix compared to $18249 \pm 45$ counts/pix outside eclipse. This corresponds to an eclipse depth of $\Delta V=5.80 \pm 0.21$. From trails of comparison stars on the same frame the brightness shortly 


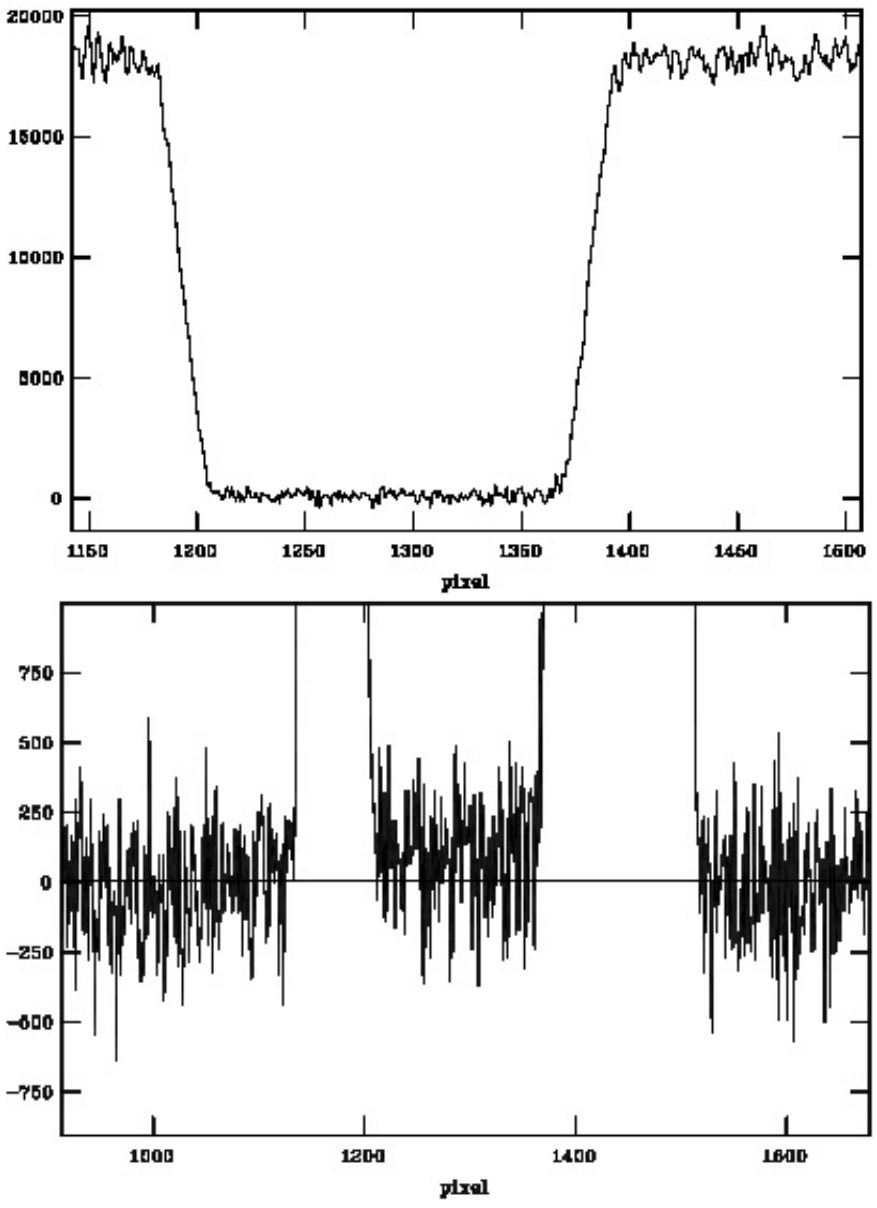

Fig. 2. Eclipse light curve in $V$ band resulting from trail observation. One pixel corresponds to a time interval of 3 seconds. Top: full eclipse curve, bottom: close-up of the signal during totality compared with zero signal.

before and after as well as during eclipse can be derived to be $V=16.98$ and 22.78, respectively. Since the light curve is perfectly flat at the bottom the eclipse is total. This allows an unambiguous determination of the length of ingress and egress to be $85 \mathrm{~s}$. Totality lasts $485 \mathrm{~s}$ and the entire eclipse duration amounts to $655 \mathrm{~s}$.

The combination of the times of mid-eclipse of the new data with those from the literature allows an update of the ephemeris. The HJDs of mid-eclipse, cycle numbers and O-C residuals are given in Table 2 . The numbers in brackets give the errors of the last digits. The mid-eclipse times given by Wood \& Marsh (1991) are based on photometry with $10 \mathrm{~s}$ (two eclipses) and $1 \mathrm{~s}$ (one eclipse) time resolution and are given to the sixth digit which seems too optimistic. Lacking any information on the quality of this photometry we adopt for these measurements error values of \pm 0.00015 which represent the order of errors we obtain from the linear regression fit. The new ephemeris based on all available data is then as follows:
Table 2. Eclipse times of NN Ser.

\begin{tabular}{lrcc}
\hline \hline $\begin{array}{l}\text { Time of minimum } \\
\text { HJD 2 } 400000+\end{array}$ & $\begin{array}{r}\text { Cycle } \\
\text { No. }\end{array}$ & $\begin{array}{c}\text { O-C } \\
\text { (days) }\end{array}$ & Reference \\
\hline $47344.524(5)$ & 0 & +0.000041 & $(1)$ \\
$47703.545090(2)$ & 2760 & +0.000001 & $(4)$ \\
$47703.675179(6)$ & 2761 & +0.000010 & $(4)$ \\
$47704.715806(3)$ & 2769 & -0.000004 & $(4)$ \\
$47705.626369(3)$ & 2776 & -0.000001 & $(4)$ \\
$47705.756461(7)$ & 2777 & +0.000000 & $(4)$ \\
$47712.78093(15)$ & 2831 & +0.000153 & $(2)$ \\
$47713.82158(15)$ & 2839 & +0.000162 & $(2)$ \\
$48301.91353(15)$ & 7360 & -0.000108 & $(2)$ \\
$51006.5398(2)$ & 28152 & +0.000316 & $(4)$ \\
$51340.71516(20)$ & 30721 & -0.000148 & $(4)$ \\
$51667.4771(4)$ & 33233 & +0.000528 & $(3)$ \\
\hline
\end{tabular}

(1) Haefner (1989); (2) Wood \& Marsh (1991);

(3) Pigulski \& Michalska (2002); (4) this paper.

\subsection{Spectroscopy}

Phase resolved spectroscopy was obtained in July 1989 and May 1990 using EFOSC at the ESO $3.6 \mathrm{~m}$ telescope and the Boller \& Chivens spectrograph at the $3.5 \mathrm{~m}$ telescope of the Calar Alto observatory. Details of these observations are given in Table 3. On-line reductions of the ESO spectra revealed the behaviour predicted on the basis of the two spectra obtained in 1988: the appearance of Balmer emissions shortly after eclipse: their phase-dependent strengthening and migration through the absorption profiles as well as their fading and disappearance shortly before eclipse. These confirm the proposed scenario of a pre-cataclysmic binary where the emission originates on the heated side of the secondary and is superimposed on the absorption of the primary. The spectra obtained in 1990 were taken with higher resolution and cover exactly two orbital phases. Integration times and phasing were chosen to be identical for both runs to enable a superposition of the corresponding spectra for better $S / N$. The spectra were reduced in the usual way using the IHAP package for those obtained on 1989 July 2 and the IRAF package for the remaining ones. Spectral features present are those already reported by Catalán et al. (1994): Balmer and He I lines, which, depending on orbital phase, appear in emission and/or absorption as well as He II $4686 \AA$ which always remains in absorption. Some selected spectra demonstrating the phase-dependent behaviour are presented in Fig. 3.

Though the single spectra in particular obtained on 1989 July 02 and 1990 May 30 are quite noisy, radial velocities could be determined for prominent emission and absorption features using multiple (if appropriate) or single Gauss approximations. The resulting heliocentric radial velocity curves are shown in Fig. 4. Note that the emission line velocity does not represent the velocity of the centre of mass of the cool star but that of the heated inner hemisphere, i.e. the true semi-amplitude must be somewhat larger than measured (see Sect. 5). 
Table 3. Journal of spectroscopic observations (time series).

\begin{tabular}{ccccccccc}
\hline \hline Date & $\begin{array}{c}\text { Start } \\
(\mathrm{UT})\end{array}$ & $\begin{array}{c}\text { Duration } \\
(\mathrm{h})\end{array}$ & $\begin{array}{c}\text { Integr. } \\
(\mathrm{m})\end{array}$ & $\begin{array}{c}\text { Dispersion } \\
(\AA / \mathrm{Am})\end{array}$ & $\begin{array}{c}\text { Resolution } \\
(\AA / \mathrm{\AA} / \mathrm{pix})\end{array}$ & $\begin{array}{c}\text { Range } \\
(\AA)\end{array}$ & $\begin{array}{c}\text { No. of } \\
\text { spectra }\end{array}$ & Telescope \\
\hline 1989 July 02 & $23: 33$ & $5: 27$ & 7 & 230 & 5.5 & $3600-7000$ & 36 & $3.6 \mathrm{~m} / \mathrm{La} \mathrm{Silla}$ \\
1989 July 03 & $23: 52$ & $0: 10$ & 10 & 120 & 3.6 & $3500-5590$ & 1 & $3.6 \mathrm{~m} / \mathrm{La} \mathrm{Silla}$ \\
1989 July 04 & $02: 53$ & $2: 43$ & 10 & 120 & 3.6 & $3500-5590$ & 15 & $3.6 \mathrm{~m} / \mathrm{La} \mathrm{Silla}$ \\
1990 May 30 & $21: 05$ & $6: 12$ & $10 / 15 / 19$ & 60 & 0.9 & $4020-4940$ & 22 & $3.5 \mathrm{~m} /$ Calar Alto \\
\hline
\end{tabular}
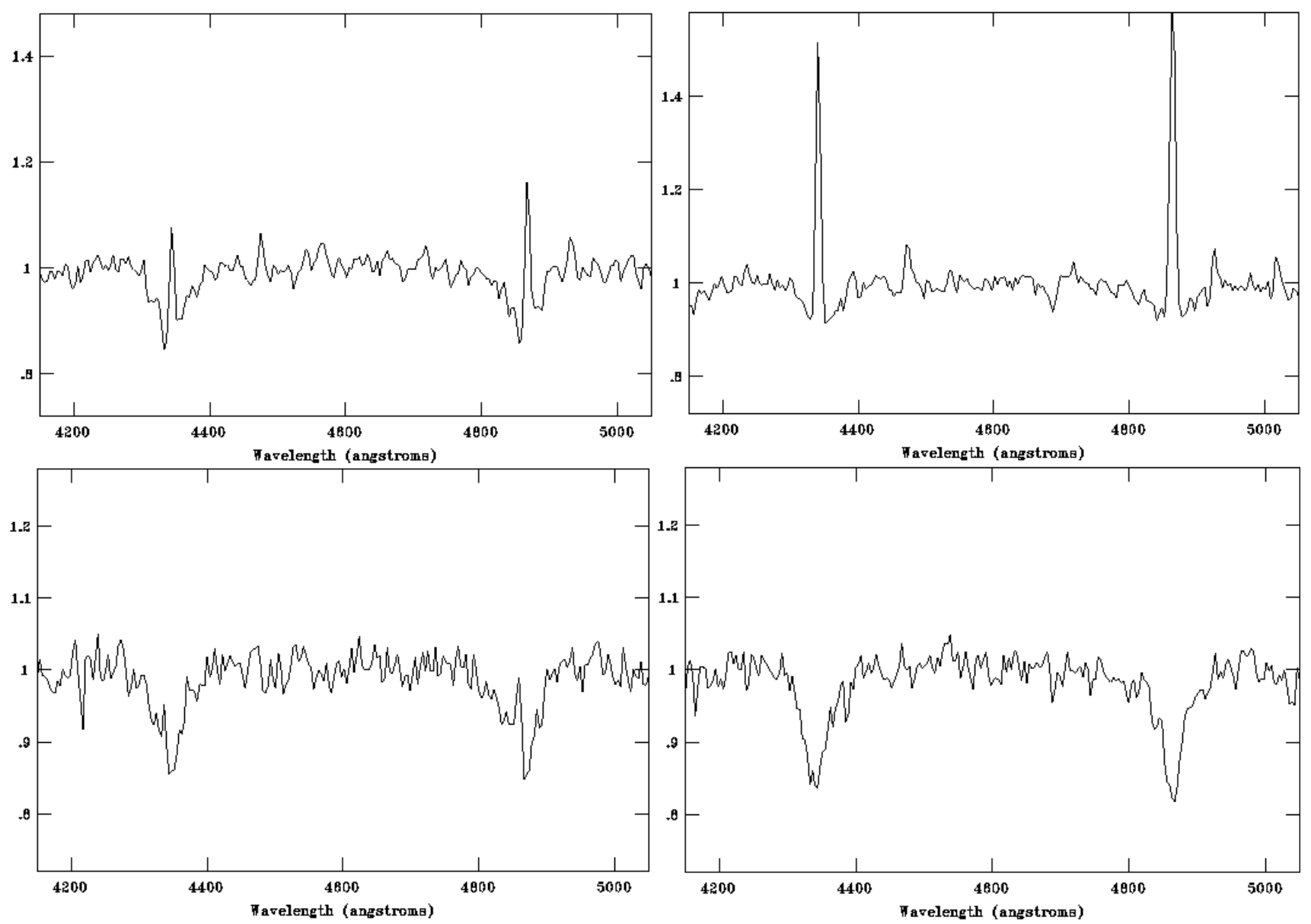

Fig. 3. Rectified spectra of NN Ser in the region $\mathrm{H}_{\beta}$ to $\mathrm{H}_{\gamma}$ taken on 1989 July 04 at different phases (top: phase 0.25 (left) and 0.47 (right), bottom: phase 0.82 (left) and 0.93 (right)) showing the phase-dependent behaviour of the emission lines. The emissions originate on the heated side of the cool star and are superimposed on the absorptions of the white dwarf.

Since it seemed extremely interesting to obtain spectral information on the secondary the FORS instruments at the VLT (FORS1 at unit 1 (ANTU), FORS2 at unit 2 (KUEYEN)) were used to record eight single spectra of the cool star during totality. To avoid any contamination by the white dwarf a single exposure time was limited to $5 \mathrm{~min}$. The spectral range covered was 5900 to $9500 \AA$ with a resolution of $5.5 \AA /$ pix. A blocking filter prevented order overlap. Though the single spectra of this 22.8 mag object were noisy (due to the faintness of the target, the short exposure time and the steadily decreasing efficiency of the CCD for wavelengths $>7000 \AA$ ), the $S / N$ of the resulting average spectrum is sufficient for spectral classification (see Sect. 3). For this purpose flux standards were observed for all nights in question. As a by-product of this spectroscopy six spectra of the white dwarf were obtained shortly before or after eclipse (see Sect. 4). Table 4 gives details for all these VLT observations.

\section{Spectral type of the secondary}

To link physical properties of the cool star with its spectral type a classification of the spectrum of the secondary in NN Ser is desirable. Despite the fact that systems like NN Ser passed through a common-envelope phase, the secondaries survived as normal main sequence stars with (after $\sim 10^{4}$ years relaxation time) nearly unaffected dimensions and composition of the surface layers (Hjellming \& Taam 1991). Further, the irradiation of the hemisphere facing the white dwarf giving rise to 


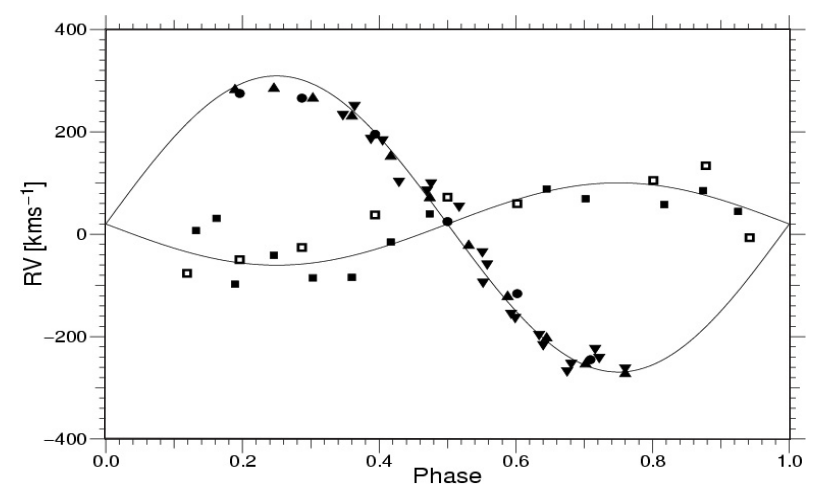

Fig. 4. Radial velocity curves of NN Ser. Emission lines: $\mathbf{v}$ : average of $\mathrm{H}_{\alpha}, \mathrm{H}_{\beta}, \mathrm{H}_{\gamma}$, and $\mathrm{H}_{\delta}$ (1989 July 02, mean error: $\pm 85 \mathrm{~km} \mathrm{~s}^{-1}$ ), $\mathbf{\Lambda}$ : average of $\mathrm{H}_{\beta}, \mathrm{H}_{\gamma}$, and $\mathrm{H}_{\delta}$ (1989 July 03/04, mean error: $\pm 80 \mathrm{~km} \mathrm{~s}^{-1}$ ), -: average of $\mathrm{H}_{\beta}, \mathrm{H}_{\gamma}$, and $\mathrm{H}_{\delta}$ (1990 May 30, mean error: $\pm 25 \mathrm{~km} \mathrm{~s}^{-1}$ ). Absorption lines: $\mathbf{\square}: \mathrm{H}_{\beta}$ (1989 July 03/04, mean error of single measurement: $\pm 30 \mathrm{~km} \mathrm{~s}^{-1}$ ), $\square: \mathrm{H}_{\gamma}$ (1990 May 30, mean error of single measurement: $\pm 40 \mathrm{~km} \mathrm{~s}^{-1}$ ). The absorption lines in the spectra obtained on 1989 July 02 were too noisy to give any reliable results. Solid lines: computed radial velocity curves using the model derived in Sect. 5. The system velocity is assumed to be $20 \mathrm{~km} \mathrm{~s}^{-1}$. Note that the observed semi-amplitude of the emission line radial velocity curve is slightly smaller than the computed K2 (see text).

Table 4. Journal of spectroscopic observations (timed single exposures) obtained at the VLT/Paranal. M: M star during eclipse of white dwarf, WD: white dwarf shortly before or after eclipse by M star.

\begin{tabular}{ccccc}
\hline \hline Date & $\begin{array}{c}\text { Start } \\
(\mathrm{UT})\end{array}$ & $\begin{array}{c}\text { Exp. time } \\
(\mathrm{m})\end{array}$ & Target & Instrument \\
\hline 1999 June 11 & $1: 55$ & 5 & $\mathrm{M}$ & FORS1 \\
1999 June 11 & $2: 04$ & 2 & WD & FORS1 \\
2000 Feb. 07 & $9: 11$ & 5 & $\mathrm{M}$ & FORS2 \\
2000 May 30 & $3: 35$ & 5 & $\mathrm{WD}$ & FORS1 \\
2000 May 30 & $3: 46$ & 5 & $\mathrm{M}$ & FORS1 \\
2000 May 30 & $6: 48$ & 1 & $\mathrm{WD}$ & FORS1 \\
2000 May 30 & $6: 53$ & 5 & $\mathrm{M}$ & FORS1 \\
2000 May 31 & $1: 29$ & 3.3 & $\mathrm{WD}$ & FORS1 \\
2000 May 31 & $1: 37$ & 5 & $\mathrm{M}$ & FORS1 \\
2000 May 31 & $4: 40$ & 1 & $\mathrm{WD}$ & FORS1 \\
2000 May 31 & $4: 44$ & 5 & $\mathrm{M}$ & FORS1 \\
2001 Feb. 26 & $6: 55$ & 5 & $\mathrm{M}$ & FORS2 \\
2001 Feb. 27 & $7: 47$ & 1 & WD & FORS2 \\
2001 Feb. 27 & $7: 53$ & 5 & $\mathrm{M}$ & FORS2 \\
\hline
\end{tabular}

chromospheric emission lines has no influence on the unirradiated part of the secondary (e.g. Smith 1995). So, spectral information on the cool star obtained during total eclipse of the white dwarf combined with other parameters (e.g. mass, radius, temperature) may serve as input for relations between fundamental properties of cool stars in general which are sometimes controversial in the literature.

The averaged spectrum of the cool star normalized to its flux at $7500 \AA$ is shown in the upper part of Fig. 5. No telluric molecular absorption features were removed. The well known molecular bands of $\mathrm{TiO}(6322,6569,7053,7666,8206,8432$, $8859 \AA$ ) and the $8190 \AA$ sodium doublet are the most prominent spectral features. VO (7851, $8521 \AA$ A) may also be present. No sign of an intrinsic activity could be detected. Colour ratios as defined by Kirkpatrick et al. (1991) to distinguish between luminosity classes were determined and revealed unambiguously the dwarf nature of the secondary in NN Ser. A morphological comparison with dwarf standards given by Kirkpatrick et al. (1991) and Steele \& Jameson (1995) hints at a spectral type of between M4.5 and M5 in the Boeshaar system. Further, to disregard the relative flux behaviour and to rely only on spectral features the spectrum was rectified by fitting a spline to the "continuum" points at 6530, 7040, 7560, 8130, 8840, and $9040 \AA$ and by dividing the spectrum by the resulting spline. The same procedure was applied to a M4.5 and a M5.0 standard taken from the Keck library of LRIS spectra of late M, L and T dwarfs. Figure 5 (lower part) shows a superposition of the three spectra indicating also a spectral type between M4.5 and M5 for the cool star in NN Ser. To use a more quantitative approach we followed methods suggested by Young \& Schneider (1981): The equivalent width of the sodium doublet and the fractional depths of the TiO bands at $7150 \AA$ and $7700 \AA$ were determined after removing the telluric features $\left(\mathrm{H}_{2} \mathrm{O}\right.$ at $8190 \AA$ and $\mathrm{O}_{2}$ at $7700 \AA$ ) by dividing the non-rectified spectrum by that of the white dwarf. A comparison of the results $\left(W_{\lambda}=7 \AA\right.$, fractional depths: 0.54 and 0.43 ) with the corresponding values of the given standards yielded spectral types of M5, M4.75 and M5 respectively. Combining and weighting all these methods we conclude that the cool star in NN Ser is of dM4.75 spectral type, to better than 0.25 . This spectral type and the temperature determined later (see Sect. 5) fit the spectral type - temperature scale derived by Brett (1995) from model atmospheres with solar abundance and $\log g=5$ nicely.

\section{Model atmosphere analysis of the primary}

Four spectra out of the 1989 July 03/04 series (Table 3) were obtained near eclipse and are thus not affected by the emissions originating on the secondary. After averaging they proved to be suitable for a model atmosphere analysis of the white dwarf as did the VLT spectra marked "WD" in Table 4. The two resulting spectra were analyzed using non-LTE model atmospheres calculated using the ALI (Accelerated Lambda Iteration) program of Kunze (1995). A grid of models including hydrogen and helium (homogeneous composition) and covering temperatures in the range of $45000 \mathrm{~K}$ to $65000 \mathrm{~K}$ and $\log g$ from 7.5 to 9.5 was computed. In addition, the helium abundance was varied from 0 . up to 0.0001 by number. The temperature/density structure of each model was then held fixed and more extensive hydrogen/helium model atoms (Husfeld et al. 1989) were used to determine the emergent flux. The $\mathrm{H} / \mathrm{He}$ populations were calculated using the DETAIL program of Butler \& Giddings (1985) as modified by Butler (priv. comm.) and the SURFACE program of the same authors provided the emergent fluxes. The latter were then compared with the observed spectrum in the region of the $\mathrm{H}_{\beta}$ line. For each gravity, those models capable of reproducing the line profile were retained, providing a locus in the $T_{\text {eff }}-\log g$ diagram. As a last step, the helium abundance was varied so as to match the observed strength of the He II line at $4686 \AA$ A. The final parameters were found to be 

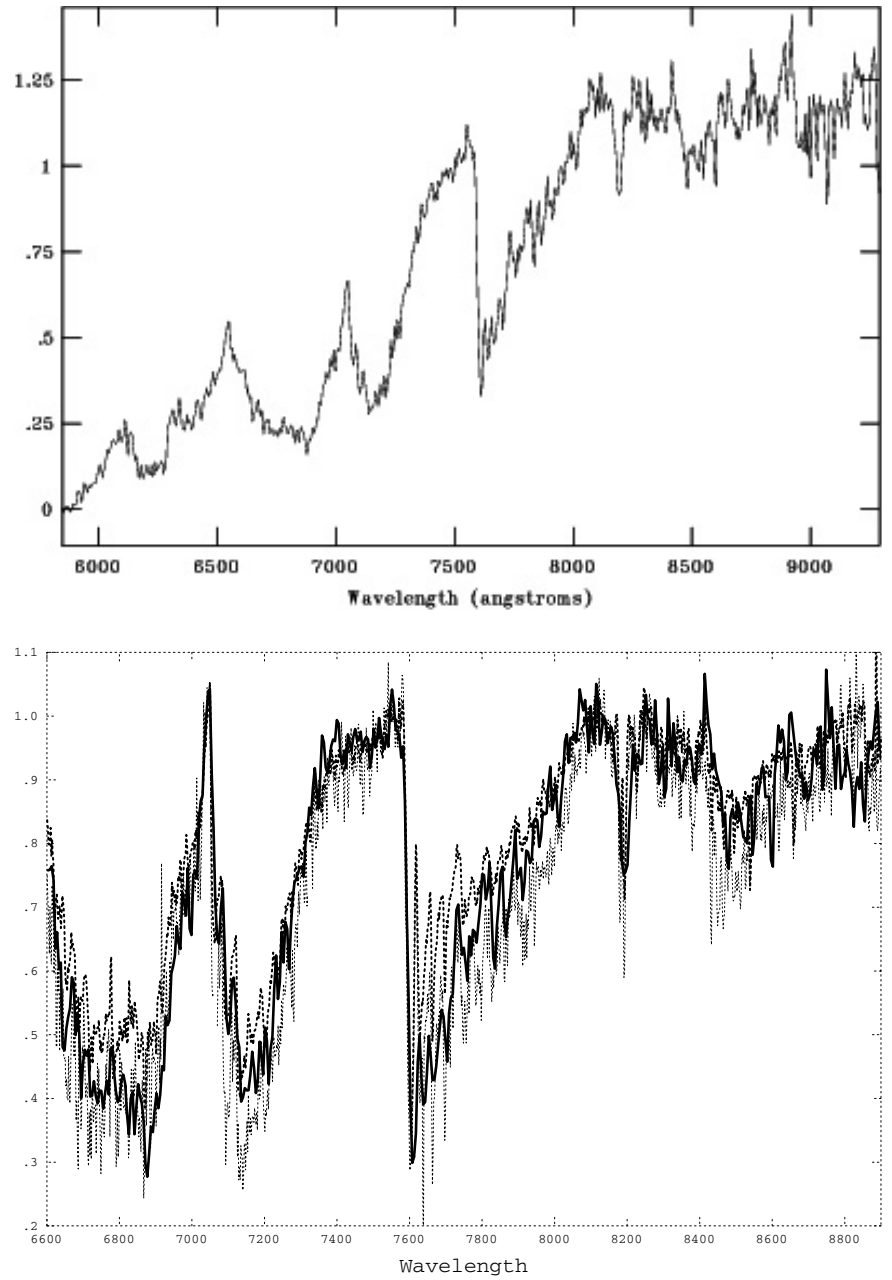

Fig. 5. Top: spectrum of the cool component in NN Ser normalized to unity at $7500 \AA$. Bottom: superposition with rectified spectra of M4.5 and M5 standards (bold line: cool component of NN Ser, dotted thick line: M4.5, dotted thin line: M5). For details see text.

$T_{\text {eff }}=57000 \pm 3000 \mathrm{~K}, \log g=7.6 \pm 0.1$ and $N_{\mathrm{He}}=2 \pm 0.5 \times 10^{-4}$ by number.

The final fit is shown in Fig. 6 and is seen to be excellent. It should be noted that neither the spectrum at shorter nor at longer wavelengths was used in the parameter determination thus providing a check on the results. Finally, we note that the lines shortward of and including $\mathrm{H}_{\delta}$ are blended to such an extent that the continuum is depressed. Thus we have defined a pseudo-continuum in this region in both the model and the observations to facilitate the comparison.

The presence of the He II line at $4686 \AA\left(W_{\lambda} \sim 300 \mathrm{~m} \AA\right)$ indicates that the white dwarf in NN Ser is a hydrogen-helium hybrid star of type DAO1 according to the classification criteria given by Sion et al. (1983). Analyzing a large sample of DAO and DA stars Bergeron et al. (1994) noticed that DAO stars show unusually low surface gravities as compared to DA stars at higher $(\gtrsim 56000 \mathrm{~K})$ temperatures. Interestingly, three stars out of the DAO sample exhibiting normal (DA) surface gravities and low $N_{\mathrm{He}}$ are members in M dwarf/white dwarf binaries. This has been taken as an indication that these stars have a different history, the helium content being due to accretion from

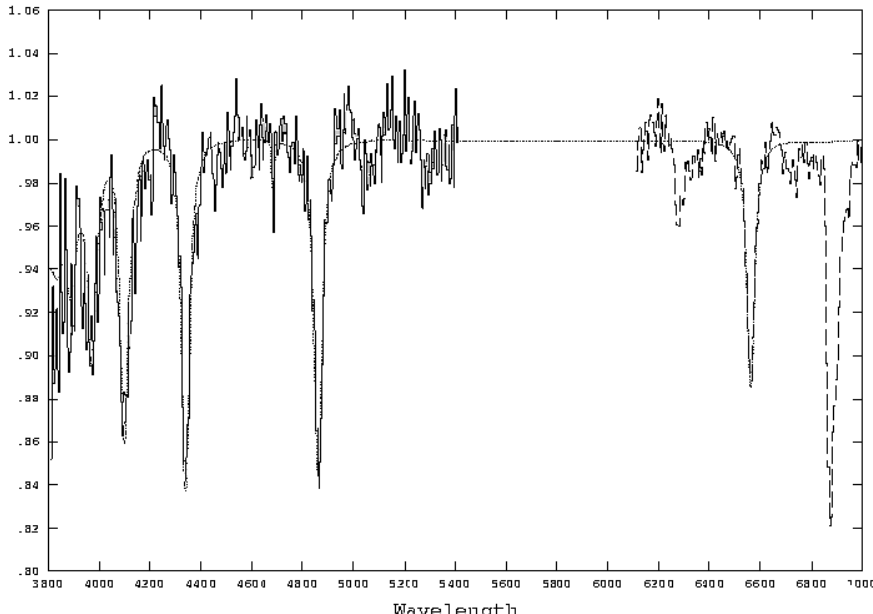

Fig. 6. Comparison of observed (full thick and dashed curves) and calculated (thin curve) spectra of the DAO1 white dwarf in NN Ser for the final parameters (see text). The continua shortward of $\mathrm{H}_{\delta}$ have been adjusted to facilitate comparison but were not used in the parameter determination.

an $\mathrm{M}$ star wind or due to interaction during the common envelope evolution rather than due to mass loss phenomena suffered by the white dwarf. The temperature, gravity and $N_{\mathrm{He}}$ values derived for the DAO star in NN Ser match those of that composite systems and, in particular, give support to weakly correlated relations found by Bergeron et al. (1994), namely, that for DAO stars, gravity is increasing with decreasing temperature and that $N_{\mathrm{He}}$ in DAOs is decreasing with increasing gravity and decreasing temperature.

The detailed mass-radius relations computed by Panei et al. (2000) for different internal compositions, surface layers and temperatures allow to draw some conclusions concerning the structure of the white dwarf in NN Ser. The observed temperature together with mass and radius derived in the following Section perfectly match the model with a carbon core surrounded by a helium layer $\left(10^{-2} M_{\odot}\right)$ and a hydrogen envelope $\left(10^{-5} M_{\odot}\right)$.

\section{Light curve analysis}

The photometric measurements were analysed using the GRADUS code which allows the geometrically and radiatively unbiased synthesis of light curves. The code was developed by Simon, Pfeiffer, and Fiedler (private communication) and has proven useful for well detached binaries (Sturm \& Simon 1994; Simon et al. 1994). It uses the standard features of Roche geometry and comprises apsidal motion, eccentric orbits and third light. The star surfaces are modelled by polyhedra of triangles allowing for a high precision approximation of their geometrical shape. The radiation is computed via model atmospheres (ATLAS9 models, Kurucz 1993) and the resulting grid of the angular and frequency dependent intensities for the required range of temperatures and gravities is stored in a table for subsequent interpolation. Limb darkening is not treated analytically but follows directly from tabulated intensities. Particular attention is focused on mutual heating of the components by means of a careful treatment of the absorption and scattering 
processes for each surface element. However, this method is applicable only to moderate illumination and fails in the case of NN Ser. The optimum fit to a given light curve (available are several standard and special filter passband systems) is found by the Downhill-Simplex relaxation procedure.

\subsection{Input parameter}

Since the system constitutes a short period binary it is safe to assume a circular orbit (which implies the absence of apsidal motion) and synchronized rotation of the components. Obviously, no third light is present. The canonical value for the radiative gravity-darkening exponent of 0.25 is applied to the white dwarf whereas a new value for the convective gravitydarkening exponent of 0.05 (Sarna 1989; Claret 2000) is used for the (low mass) M star. In determining the mass ratio we do not rely on the radial velocity curves (Fig. 4) since the velocity curve of the white dwarf is of very low quality and that of the $\mathrm{M}$ star does not represent the true velocity, i.e the velocity of its centre of mass. The corresponding correction can only be estimated iteratively assuming or deriving special intensity distributions over the illuminated hemisphere in combination with further system parameters. Therefore, we determine the mass of the $\mathrm{M}$ star $\left(0.150 \pm 0.008 M_{\odot}\right)$ iteratively using the radii resulting from fits to the eclipse light curve and the empirical mass-radius relation given by Ségransan et al. (2003). This relation is mainly based on radii measured interferometrically using the VLTI and the PTI and on masses inferred from IR photometry. It is in excellent agreement with the theoretical models given by Baraffe et al. (1998). Further, the mass of the white dwarf is determined via a recently established gravity/temperature diagram for hot white dwarfs (Napiwotzki et al. 1999) which also includes evolutionary tracks calculated by Blöcker (1995). With our derived values for the white dwarf's temperature $(57000 \mathrm{~K}$, which is kept constant in the analysis) and gravity $(\log g=7.6)$ we obtain $0.54 \pm 0.05 M_{\odot}$ for its mass. Test runs showed that the surfaces of the white dwarf and the M star are best approximated by 3968 and 9800 triangles, respectively. Only the most reliable MCCP measurements (1989 June 27) and the VLT observations are used for the following light curve analysis.

\subsection{Results}

With the input parameters derived above we first analysed the eclipse light curve ( $V$ band) to derive the radii of the components, the inclination of the system, the binary separation, and the temperature of the un-heated side of the M star. As a byproduct the gravities and radial velocity semi-amplitudes are calculated. Since the M star contributes less than $1 \%$ to the flux in this part of the light curve it is sufficient to use black body intensities for the cool component. As long as the eclipsing star is of spherical shape no information on its limb-darkening is required because only the normalized flux enters the calculation. Although the light curve analysis revealed a marginally ellipsoidal shape, it turned out that this can, in fact, be neglected in this respect. The angular dependent intensities for
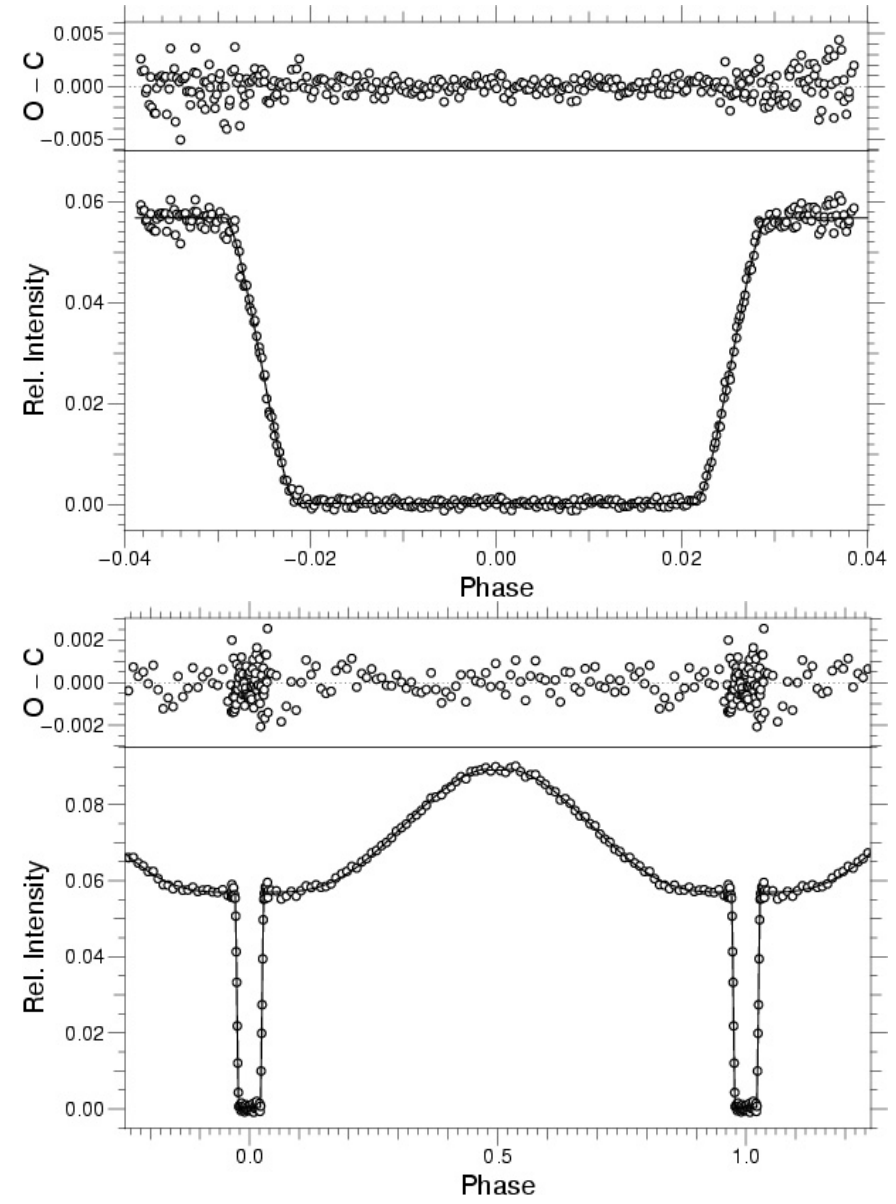

Fig. 7. The observed (circles) and synthetic (solid line) light curve of NN Ser ( $V$ band, trail data combined with MCCP data of 1989 June 27). Top: close-up of eclipse (measurements are binned to $3 \mathrm{~s}$ and $6 \mathrm{~s}$ resolution for the trail and the MCCP data, respectively, to get approximately the same $S / N$ of $\sim 35$ ), bottom: complete light curve (the measurements are binned to $110 \mathrm{~s}$ resolution outside eclipse and to $12 \mathrm{~s}$ resolution during eclipse). Top panels: residuals of the fits.

the white dwarf are calculated via model atmospheres and interpolated from an appropriate table. By evaluating the synthetic eclipse curve we found a strong correlation between the inclination and the radii of the components in the sense that an increasing inclination is compensated by an increasing radius of the white dwarf and an decreasing radius of the $\mathrm{M}$ star (and vice versa) without significant change in the quality of the fit. The upper limit of the inclination is set when the quality of the eclipse fit diminishes and at the same time a secondary (not observed) eclipse appears, which is caused by the occultation of a larger and hotter area on the $\mathrm{M}$ star. The lower limit is found when the white dwarf exhibits a gravity in excess of that determined spectroscopically. Finally we arrived at the following parameters which best represent the observed eclipse curve (see Fig. 7 upper part): inclination: $84.6^{\circ} \pm 1.1^{\circ}$, binary separation: $0.9543 \pm 0.0233 R_{\odot}$, radius of white dwarf: $0.189 \pm$ $0.0010 R_{\odot}$, radius of $\mathrm{M}$ star (polar): $0.174 \pm 0.009 R_{\odot}$, temperature of M star (backside): $2920 \pm 70 \mathrm{~K}$. As already mentioned, the shape of the M star turned out to be slightly non-spherical. 
Table 5. Coefficients for limb-darkening and heating as derived from the two simultaneous fits to the VRI light curves. For details see text.

\begin{tabular}{ccccc}
\hline \hline$T$ (sub-st. point) & $u_{1}$ & $u_{2}$ & $h_{1}$ & $h_{2}$ \\
\hline $7125.3 \mathrm{~K}$ & $-1.1268(V)$ & - & 0.3848 & 0.0088 \\
& $-1.1330(R)$ & - & & \\
$7125.5 \mathrm{~K}$ & $-1.2302(I)$ & - & & \\
& $-1.1156(V)$ & -0.0099 & 0.3849 & 0.0081 \\
& $-1.0973(R)$ & -0.0383 & & \\
& $-1.1544(I)$ & -0.0840 & & \\
\hline
\end{tabular}

The corresponding radii are given in Table 6 which also includes the derived gravities and radial velocity semiamplitudes.

Modelling the pronounced reflection effect in NN Ser is not a straightforward task. Since our knowledge concerning the complex physical processes in a highly irradiated atmosphere is still limited, no full treatment of the problem exists to date. Vaz \& Nordlund (1985), Nordlund \& Vaz (1990) and Alencar \& Vaz (1999) investigated the reflection effect using LTE model atmospheres where the temperature differences of the involved stars were in the order of up to $8000 \mathrm{~K}$. The intention was to derive values for the bolometric reflection albedo and analytical expressions for the limb-darkening coefficients for use with light curve synthesis programs. Non-LTE techniques were applied by e.g. Proga et al. (1996) and by Pustylnik et al. (2001) to model the structure of the outer layers of strongly irradiated atmospheres (temperature differences $\gtrsim 40000 \mathrm{~K}$ ).

Emergent synthetic spectra for NN Ser (white dwarf and heated part of $\mathrm{M}$ star) were calculated by Sakhibullin \& Shimansky (1996) and compared with those observed. They kept the assumed system separation $\left(0.95 R_{\odot}\right)$ and the assumed masses $\left(0.57\right.$ and $0.12 M_{\odot}$ for white dwarf and $\mathrm{M}$ star, respectively) fixed and found the following system parameters: inclination: $82^{\circ}$, white dwarf: radius: $0.019 R_{\odot}$, temperature: $3500 \mathrm{~K}, \mathrm{M}$ star: radius: $0.20 R_{\odot}$, temperature: $62000 \mathrm{~K}$. Though these results differ considerably from those obtained in this paper they nevertheless constitute a first attempt to quantitatively understand the spectra formed on the heated side of the M star in NN Ser.

Given the lack of a sound physical basis for the pronounced reflection effect of NN Ser we have tried to match the out-ofeclipse light curve by adjusting the albedo and limb-darkening coefficient of the heated side of the M star. Such a procedure has already been applied in similar cases (e.g. Hilditch et al. 1996). Moreover, in several test runs we probed different limbdarkening laws (linear, quadratic, cubic) and different assumptions concerning the albedo (constant, linear, quadratic or cubic functions of the local temperature), while keeping all the parameters derived from the fit to the eclipse light curve fixed. The temperature on the irradiated side is calculated for each surface element from the equation $T_{\text {hot }}^{4}=T_{2}^{4}+g * h * T_{1}^{4}$, where $T_{1}$ and $T_{2}$ are the temperatures of white dwarf and $\mathrm{M}$ star, respectively. The geometric factor $g=\rho^{2} * \cos \phi$ is always smaller than 0.0007 , with $\rho$ being the ratio between the radius of the white dwarf and its distance to the M star's surface element in question and $\phi$ being the incidence angle. The heating factor $h=1-a$, with $a$ being the bolometric albedo, describes the thermalization of the incident radiation. Possible horizontal energy transfer due to temperature differences in adjacent surface elements and due to coriolis forces is not considered. The results of the test runs ( $V$ band) revealed that the light curve can be matched yielding temperatures in the range of 6900 to $7200 \mathrm{~K}$ (sub-stellar point) for the various limb-darkening and albedo relations mentioned above. Interestingly, the value for the constant term of the limb-darkening coefficient is always close to -1.15 (i.e. we are actually dealing with limbbrightening) and that of the albedo close to 0.6 , irrespective of which combination and which higher order approximation of the different laws is used. Thus the limb-darkening coefficient is considerably different from that expected for a non-irradiated convective star with a temperature around $3000 \mathrm{~K}$, namely 0.7 (Díaz-Cordovés et al. 1995), whereas the albedo turns out to be near the canonical value of 0.5 . In fitting the remaining light curves we confined ourselves to a quadratic limb-darkening relation $\left(1-u_{1}(1-\mu)-u_{2}(1-\mu)^{2}\right.$ with $\mu=\cos \theta$ and $\theta$ being the line-of-sight angle) in combination with a constant value or a linear expression $\left(h=h_{1}+h_{2} *\left(T_{\mathrm{hot}}-6000\right) / 1000\right)$ for the heating factor. This expression tries to account for a temperature dependency of $\mathrm{h}$, where the normalizing factor is introduced by the finding that areas with temperatures below $5000 \mathrm{~K}$ contribute less than $10 \%$ to the intensity (in $V$ band). Applying the linear expression requires, of course, an iterative solution in calculating $T_{\text {hot }}$. Whereas excellent fits to the $V, R$, and $I$ light curves could be achieved yielding temperatures around $7200 \mathrm{~K}$ and almost identical values for the limb-darkening coefficients and the albedo, those in the $B$ and especially in the $U$ band were of low quality, probably due to the crowded Balmer emissions which influence the measurements in these bands and which are not considered in our black body approximation. Therefore, we concentrated on the VRI measurements only and obtained from a simultaneous fit to the light curves a temperature of $7125 \mathrm{~K}$ for the sub-stellar point with an estimated error of $\pm 200 \mathrm{~K}$. The corresponding coefficients for limb-darkening and heating factor are compiled in Table 5. The fit to the $V$ light curve is provided in Fig. 7 (lower part). The resulting temperature distribution over the heated side is shown in Fig. 8 and is believed to be a fair approximation to the real conditions despite the fact that no dynamical effects were taken into consideration. Table 6 summarizes all observed and derived system parameters of NN Ser. A new distance estimate of $500 \pm 35$ pc is also given which is based on the white dwarf's temperature, radius and brightness $(V \sim 17)$, a bolometric correction of 4.92 in $V$ (Bergeron et al. 1995) and $E_{B-V}=0.05($ Wood \& Marsh 1991).

\section{Conclusion}

Though the number of known pre-cataclysmic binaries has increased over the past few years, the catalogue by Ritter \& Kolb (2003) lists only 17 white dwarf-M star systems with more or less well estimated parameters. Of these only four systems are eclipsing and offer an opportunity for a more precise parameter determination. Among these NN Ser is an outstanding example. It harbours the hottest white dwarf, shows the most 

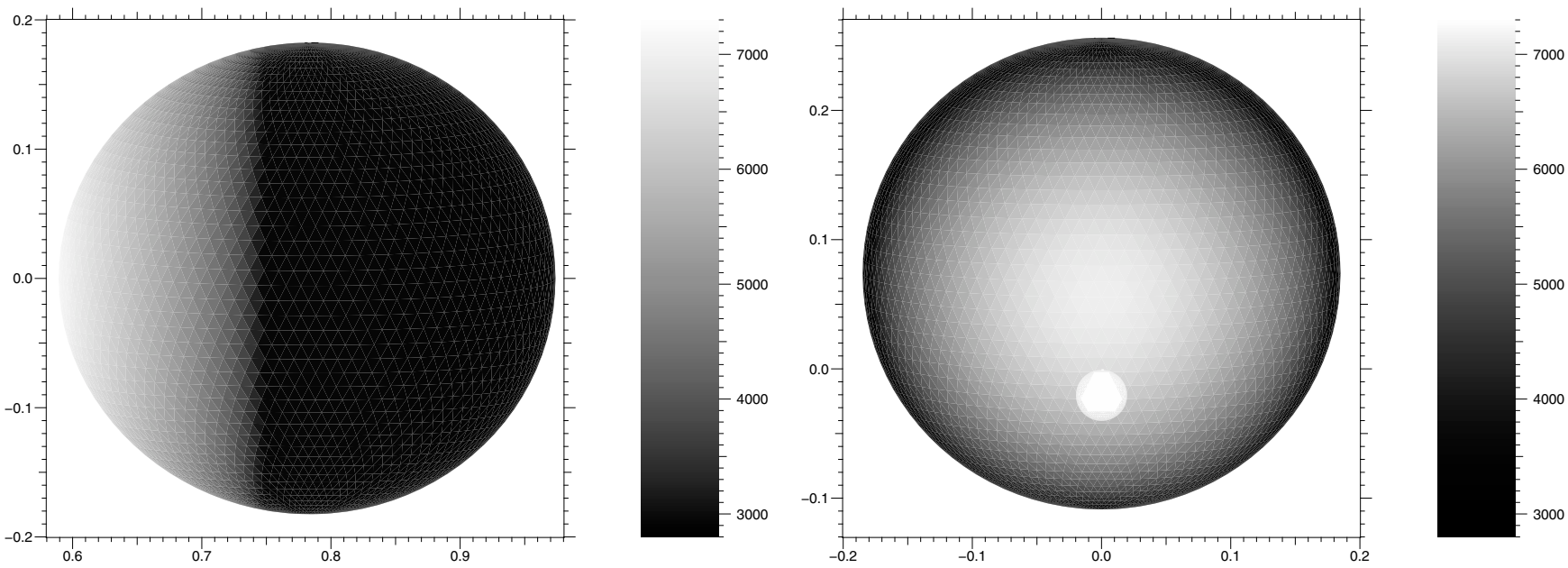

Fig. 8. The temperature distribution over the surface of the $M$ star, left: seen at phase 0.25 , right: seen at phase 0.50 . The axes are in units of the binary separation. The origin of co-ordinate system is located in the centre of gravity. The grey scales give the temperature ranges, respectively. The temperature of the white dwarf in front of the M star (right) is outside the gray scale range.

Table 6. System parameters of NN Ser. WD: white dwarf, RD: red dwarf, RD radius/temperature: pol: polar, sub: sub-stellar point, back: backside, side: perpendicular to pol/sub/back, $\log g_{m a}: \log g$ from model atmosphere analysis, $\log g_{l c}: \log g$ from light curve analysis.

\begin{tabular}{lll}
\hline \hline & Catalán et al. (1994) & This paper \\
& Wood \& Marsh $(1991)$ & \\
\hline Period (days) & $0.130080094(23)$ & $0.1300801195(45)$ \\
Inclination & $84^{\circ}<i<90^{\circ}$ & $84.6^{\circ} \pm 1.1^{\circ}$ \\
Binary separation & $0.95 \pm 0.025 R_{\odot}$ & $0.9543 \pm 0.0233 R_{\odot}$ \\
Mass ratio & $0.18<\mathrm{q}<0.23$ & $0.2778 \pm 0.0297$ \\
WD mass & $0.57 \pm 0.04 M_{\odot}$ & $0.54 \pm 0.05 M_{\odot}$ \\
RD mass & $0.10<M_{\odot}<0.14$ & $0.150 \pm 0.008 M_{\odot}$ \\
WD radius & $0.017<R_{\odot}<0.021$ & $0.0189 \pm 0.0010 R_{\odot}$ \\
RD radius pol & $0.15<R_{\odot}<0.18$ & $0.174 \pm 0.009 R_{\odot}$ \\
RD radius sub & - & $0.185 \pm 0.009 R_{\odot}$ \\
RD radius back & - & $0.182 \pm 0.009 R_{\odot}$ \\
RD radius side & - & $0.177 \pm 0.009 R_{\odot}$ \\
WD log $g_{\text {ma }}$ & - & $7.6 \pm 0.1$ \\
WD log $g_{\text {lc }}$ & - & $7.617 \pm 0.062$ \\
RD log $g_{\text {lc }}$ pol & - & $5.141 \pm 0.051$ \\
WD temperature & $55000 \pm 8000 \mathrm{~K}$ & $57000 \pm 3000 \mathrm{~K}$ \\
RD temp. pol & $2900 \pm 150 \mathrm{~K}$ & $2950 \pm 70 \mathrm{~K}$ \\
RD temp. back & - & $2920 \pm 70 \mathrm{~K}$ \\
RD temp. side & - & $2940 \pm 70 \mathrm{~K}$ \\
RD temp. sub & $5650<T<8150 \mathrm{~K}$ & $7125 \pm 200 \mathrm{~K}$ \\
K1 & - & $80.4 \pm 4.1 \mathrm{~km} \mathrm{~s}^{-1}$ \\
K2 & $310 \pm 10 \mathrm{kms}^{-1}\left(\mathrm{H}_{\alpha}\right)$ & $289.3 \pm 12.9 \mathrm{~km} \mathrm{~s}^{-1}$ \\
WD type & $\mathrm{DAO} 1$ & $\mathrm{DAO} 1$ \\
RD spectral type & $\mathrm{M} 4.7-\mathrm{M} 6.1$ & $\mathrm{M} 4.75 \pm 0.25$ \\
Distance & $356 \mathrm{pc}<d<472 \mathrm{pc}$ & $500 \pm 35 \mathrm{pc}$ \\
\hline
\end{tabular}

pronounced reflection effect, exhibits the shortest and one of the deepest eclipses, and represents the smallest system by dimension. Based on new and unpublished older photometric and spectroscopic measurements we have been able to derive a selfconsistent model for NN Ser and to improve nearly all observed and derived system parameters.
The history and fate of 30 post common envelope binaries has recently been estimated by Schreiber \& Gänsicke (2003). According to these authors NN Ser arrived at the main sequence some $4.4 \times 10^{8}$ years ago. At that time the mass of the white dwarf progenitor was about $3 M_{\odot}$. The time elapsed after the end of the common envelope phase (cooling time of the white dwarf) amounts to about $1.3 \times 10^{6}$ years. In about $2 \times$ $10^{9}$ years (loss of angular momentum due to magnetic braking) the system will arrive at a semi-detached configuration and start the mass transfer, i.e. the activity of a cataclysmic binary. At that time the temperature of the white dwarf will have cooled to about $7000 \mathrm{~K}$ and the orbital period shortened to about $1^{\mathrm{h}} 45^{\mathrm{m}}$. Thus, since NN Ser will reach this status within a Hubble-time, the system belongs to the group of post common envelope binaries which is representative of the progenitors of the current population of cataclysmic binaries.

The present system parameters can only be improved by the acquisition of better spectroscopic material. We hope that this almost unique system will be the subject of such observations in the future.

Acknowledgements. We are indebted to B. Muschielok and R. Mendez for taking the VLT spectra on 2000 Feb. 07 resp. May 30/31 and to $\mathrm{H}$. Fiedler for performing the photometric measurements on 1998 July 12.

\section{References}

Alencar, S. H. P., \& Vaz, L. P. R. 1999, A\&AS, 135, 555

Appenzeller, I., Fricke, K., Fürtig, W., et al. 1998, The Messenger, 94, 1

Baraffe, I., Chabrier, G., Allard, F., \& Hauschildt, P. H. 1998, A\&A, 337,403

Barwig, H., Schoembs, R., \& Buckenmayer, C. 1987, A\&A, 175, 327

Bergeron, P., Wesemael, F., Beauchamp, A., et al. 1994, ApJ, 432, 305 Bergeron, P., Wesmael, F., \& Beauchamp, A. 1995, PASP, 107, 1047

Blöcker, T. 1995, A\&A, 299, 755

Brett, J. M. 1995, A\&A, 295, 736 
Butler, K., \& Giddings, J. 1985, in Newsletter on Analysis of Astronomical Spectra, Vol. 9, Daresbury Laboratory

Catalán, M. S., Davey, S. C., Sarna, M. J., Smith, R. C., \& Wood, J. H. 1994, ApJ, 269, 879

Claret, A. 2000, A\&A, 359, 289

Díaz-Cordovés, J., Claret, A., \& Giménez, A. 1995, A\&AS, 110, 329

Haefner, R. 1989, A\&A, 213, L15

Haefner, R. 2000, The Messenger, 100, 42

Hjellming, M. S., \& Taam, R. E. 1991, ApJ, 370, 709

Hilditch, R. W., Harries, T. J., \& Hill, G. 1996, MNRAS, 279, 1380

Husfeld, D., Butler, K., Heber, U., \& Drilling, J. S. 1989, A\&A, 222, 150

Kirkpatrick, J. D., Henry, T. J., \& McCarthy, D. W. 1991, ApJS, 77, 417

Kunze, D. 1995, Ph.D. Thesis, Ludwig-Maximilians-Universität Munich

Kurucz, R. L. 1993, Kurucz CD-ROM No. 13, Smithsonian Astrophysical Observatory, Cambridge, Mass.

Napiwotzki, R., Green, P. J., \& Saffer, R. A. 1999, ApJ, 517, 399

Nordlund, Å., \& Vaz, L. P. R. 1990, A\&A, 228, 231

Panei, J. A., Althaus, L. G., \& Benvenuto, O. G. 2000, A\&A, 353, 970

Pigulski, A., \& Michalska, G. 2002, IBVS, No. 5218
Proga, D., Kenyon, S. J., Raymond, J. C., \& Mikołajewska, J. 1996, ApJ, 471, 930

Pustylnik, I., Pustynski, V., \& Kubat, J. 2001, Odessa Astron. Publ., 14,87

Ritter, H., \& Kolb, U. 2003, A\&A, 404, 301

Sakhibullin, N. A., \& Shimansky, V. V. 1996, Odessa Astron. Publ., 9, 94

Sarna, M. J. 1989, A\&A, 224, 98

Schreiber, M., \& Gänsicke, B. T. 2003, A\&A, 406, 305

Ségransan, D., Kervella, P., Forveille, T., \& Queloz, D. 2003, A\&A, 397, L5

Smith, R. C. 1995, in ASP Conf. Ser. 85, ed. D.A.H. Buckley \& B. Warner, 417

Steele, I. A., \& Jameson, R. F. 1995, MNRAS, 272, 630

Sturm, E., \& Simon, K. P. 1994, A\&A, 282, 93

Simon, K. P., Sturm, E., \& Fiedler, A. 1994, A\&A, 292, 507

Sion, E. M., Greenstein, J. L., Landstreet, J. D. et al. 1983, ApJ, 269, 253

Vaz, L. P. R., \& Nordlund, Å. 1985, A\&A, 147, 281

Wood, J. H., \& Marsh, T. R. 1991, ApJ, 381, 551

Young, P., \& Schneider, D. P. 1981, ApJ, 247, 960 